Check for updates

Cite this: RSC Adv., 2019, 9, 21903

Received 3rd April 2019

Accepted 9th July 2019

DOI: 10.1039/c9ra02479d

rsc.li/rsc-advances

\section{Effect of silane functionalized graphene prepared by a supercritical carbon dioxide process on the barrier properties of polyethylene terephthalate composite films}

\author{
Jiajia Wei, ${ }^{a b}$ Shigui Peng, ${ }^{\text {ab }}$ Bin Xue, ${ }^{b}$ Zhao Yang, ${ }^{b}$ Shuhao Qin, ${ }^{\text {ab }}$ Jie Yu ${ }^{\text {ab }}$ \\ and Guomin Xu (DD *ab
}

In this work, a simple and eco-friendly strategy to modify graphene nanoplatelets (GNs) with different silane coupling agents using a supercritical carbon dioxide $\left(\mathrm{Sc}-\mathrm{CO}_{2}\right)$ process has been presented, and effect of the modified GNs on the oxygen transmission rate (OTR) and water vapor transmission rate (WVTR) of GN/PET composite films was studied. FT-IR, SEM, EDX and TG results indicated that $\mathrm{Sc}-\mathrm{CO}_{2}$ process was an effective strategy to modify GNs with silane coupling agents. Addition of the modified GNs into PET matrix could greatly decrease the OTR and WVTR values of the GNs/PET composite films, and the WVTR of GNs560/PET composite film and OTR of GNs550/PET composite film were respectively decreased about $90.08 \%$ and $58.04 \%$, as compared to those of GNs/PET composite film. It is found that the gas barrier property of GN/PET composites was attributed to not only the tortuous path effect caused by GNs themselves and the interfacial interaction, but also the affinity of binding bonds between GNs and the polymer to the gas molecules. It is believed that this work provided a strategy to design and prepare $\mathrm{CN} /$ polymer composites with high barrier properties.

\section{Introduction}

Polyethylene terephthalate (PET) is widely used in packaging, electronic devices, and solar photovoltaic cells, etc., due to its versatile properties, such as low cost, excellent impact resistance, chemical resistance, transparency, and good barrier properties. $^{\mathbf{1 , 2}}$ Unfortunately, the water vapor transmission rate (WVTR) and oxygen transmission rate (OTR) of the commonly used PET films usually fail to meet the requirements of the above applications. In fact, there is still a significant gap in gas barrier performance in these fields.

Several attempts have been made to improve the barrier properties of polymer films in recent years. Among them, addition of graphene nanoplatelets (GNs) into polymers has generated a lot of interest, owing to the excellent barrier properties and transparency of GNs. ${ }^{3-5}$ Studies have shown that the barrier properties of GN/polymer films were determined by the dispersion structure of GNs and the interfacial adhesion between GNs and polymer matrix. ${ }^{6,7}$ According to recent studies, modification of GNs with silane coupling agents was one of the effective ways to improve its dispersibility and interfacial

${ }^{a}$ Department of Polymer Material and Engineering, College of Materials and Metallurgy, Guizhou University, Guiyang 550025, China. E-mail: 410034801@qq.com ${ }^{b}$ National Engineering Research Center for Compounding and Modification of Polymer Materials, Guiyang, 550014, China adhesion between GNs and polymer matrix. Xu et al. ${ }^{8}$ found that modification of GO with 3-amino propyltriethoxy silane (APTS) in solution could improve the dispersibility and the bridging action between GO and polybenzoxazine (PBZ) resin, resulting in good thermal resistance and strong mechanical properties of the composites. While Rashidi A. et al. ${ }^{9}$ reported that the interfacial interactions between GO and epoxy was also enhanced greatly by modifying GO with (3-aminopropyl)triethoxysilane, and the pull-off adhesion strength of epoxy composite coatings to substrate was enhanced about two times. However, it is noteworthy that the common surface modification technology of GNs was solution processing, which usually involves organic solvents, making the process is difficult to implement widely for the eco-unfriendly solvent.

Recently, modification of layered nanoparticles with supercritical carbon dioxide $\left(\mathrm{Sc}-\mathrm{CO}_{2}\right)$ has attracted widely attention. It is found that $\mathrm{Sc}-\mathrm{CO}_{2}$ processing can tune not only the surface property of the nanoparticles, but also the size of the nanoparticle aggregates, improving the dispersion of layered nanoparticles in polymer matrix, enhancing the interfacial interactions between layered nanoparticles and polymer matrix. ${ }^{10,11}$ In fact, $\mathrm{Sc}_{-} \mathrm{CO}_{2}$ processing was an effective and eco-friendly technology to modify layered nanoparticles, which could overcome some drawbacks of conventional solution process.

Although silane modified montmorillonite could be obtained by $\mathrm{Sc}-\mathrm{CO}_{2}$ processing according to previous 
literatures, ${ }^{12-15}$ to the best of our knowledge, modification of GNs with silane coupling agents by $\mathrm{Sc}-\mathrm{CO}_{2}$ processing has not been reported so far. Therefore, in the present study, $\mathrm{Sc}-\mathrm{CO}_{2}$ process was employed to modify GNs with different silane coupling agents. Then, the modified GNs were blended with PET by a multistage stretching extrusion process. The structure and morphology of the modified GNs were characterized by FTIR, SEM-EDX and TG analysis, and the microstructures and final barrier property of GN/PET composites were also studied systematically, but our efforts were focused on studying effect of the structure of silane coupling agents on barrier property of GN/PET composite films and revealing the barrier mechanism of GN/PET composites. We hope that this work can pave a way for improving the barrier properties of GN/polymer composites.

\section{Experimental}

\subsection{Materials}

PET pellets (CB602) were purchased from Far Eastern Industries (Shanghai). GNs (layer number of 5 to 6 , flake diameter of $100 \mathrm{~nm}$ to $3 \mu \mathrm{m}$, and specific surface area greater than $400 \mathrm{~m}^{2}$ $\mathrm{g}^{-1}$ ) was provided by Shanxi Institute of Coal Chemistry, Chinese Academy of Sciences. $\gamma$-Aminopropyl triethoxysilane (KH550), $\gamma$-glycidyloxy propyl trimethoxysilane (KH560), and $\gamma$ methacryloxy propyl trimethoxysilane (KH570) were obtained from Zhongjie Chemical Technology Company (Guangzhou). Ethanol and acetic acid were purchased from Sinopharm Chemical Reagent Company. Carbon dioxide $\left(\mathrm{CO}_{2}\right)$ was obtained from Air Liquide, (Guiyang, China) (purity 99.9\%).

\subsection{Surface modification of GNs by $\mathrm{Sc}-\mathrm{CO}_{2}$ process}

Firstly, $10 \mathrm{~g}$ of silane coupling agent (KH550 or KH560 or $\mathrm{KH} 570$ ) was hydrolyzed in $20 \mathrm{~g}$ solution mixture of anhydrous ethanol and distilled water (mass ratio of $9: 1$ ) at $\mathrm{pH} 4.0$ for $2 \mathrm{~h}$. Then the hydrolyzed mixture and $1 \mathrm{~g}$ GNs were taken in a highpressure vessel. The mixture was processed in $\mathrm{Sc}_{-} \mathrm{CO}_{2}$ for $2 \mathrm{~h}$ at $40{ }^{\circ} \mathrm{C}$ and $20 \mathrm{MPa}$ under constant stirring. Finally, the vessel was depressurized to ambient conditions rapidly and the obtained GNs was washed with ethanol five times at room temperature, and then dried at $60^{\circ} \mathrm{C}$ for $12 \mathrm{~h}$. The samples after post-processing were correspondingly designated as GNs550, GNs560, and GNs570.

\subsection{Preparation of GN/PET nanocomposites}

Before melting process, PET was dried at $150{ }^{\circ} \mathrm{C}$ for $5 \mathrm{~h}$ and GNs were dried at $60{ }^{\circ} \mathrm{C}$ for $12 \mathrm{~h}$, in order to reduce moisture-induced thermal degradation. Then, PET pellets and $0.03 \mathrm{wt} \%$ pristine GNs or modified GNs were mixed thoroughly in a mixing bag and subsequently were molded using a multistage stretching extrusion system, as shown in ref. 16. The set-up included a two-screw extruder, a connector, and five laminating-multiplying elements (LMEs). After cooling, a $0.6 \pm 0.1 \mathrm{~mm}$ thick and $20 \pm 5 \mathrm{~mm}$ wide GN/PET composite film was formed. The five heating zones of the two-screw extruder were set to $180{ }^{\circ} \mathrm{C}, 230^{\circ} \mathrm{C}, 255^{\circ} \mathrm{C}, 260{ }^{\circ} \mathrm{C}$, and $260{ }^{\circ} \mathrm{C}$ and the screw speed was $200-250 \mathrm{rpm}$. The temperatures of LMEs were set to $260^{\circ} \mathrm{C}, 260^{\circ} \mathrm{C}, 260^{\circ} \mathrm{C}$, and $260^{\circ} \mathrm{C}$ and those of die were set to $260{ }^{\circ} \mathrm{C}, 255{ }^{\circ} \mathrm{C}$, and $255{ }^{\circ} \mathrm{C}$. The obtained composite sheets were correspondingly marked as GN/PET, GNs550/PET, GNs560/PET, and GNs570/PET. For comparison, neat PET sheet was also prepared under the same processing conditions as mentioned above.

\subsection{Characterization}

The surface functional groups of GNs were identified by Fourier transforms infrared spectrometer (FT-IR, NEXUS 570, Nicolet, USA). All the samples were pressed into $\mathrm{KBr}$ pellets. Scanning electron microscopy (SEM, Quanta FEG250, FEI, USA) was performed to observe the morphology of GNs and the energy dispersive X-ray analyzer (EDX, INCA-350, Oxford, UK) was employed to determine the elemental composition of GNs surface.

The grafted ratio of silane coupling agents on GNs surface was analyzed by thermogravimetric analyzer (TG, Q50, TA Instruments, USA), and that was calculated by following equations:

$$
\begin{aligned}
& \text { Grafted amount }\left(\text { mequiv } \mathrm{g}^{-1}\right)=\frac{10^{3} W_{1}}{\left(100-W_{1}\right) M} \\
& \text { Grafted ratio }(\%)=\frac{[\text { Grafted amount }]}{[C]} \times 100 \%
\end{aligned}
$$

where $W_{1}$ indicates the weight loss of GNs in the temperature range from $200{ }^{\circ} \mathrm{C}$ to $700{ }^{\circ} \mathrm{C}, M$ is molecular weight of silane coupling agent grafting onto the surface GNs; $C$ is the feed ratios of silane coupling agent, representing the amount of silane coupling agent in millimoles per gram of GNs.

WVTR and OTR of the prepared composite films were determined using water vapor transmission tester (PERMATRAN-W 3/33, MOCON, USA) at room temperature with $100 \%$ relative humidity and oxygen transmission tester (OxTran Model 2/21, MOCON, USA) at room temperature, respectively.

The dispersion structure of the prepared CN/PET composite films was studied by a transmission electron microscope (TEM, JEM-200CX, JEOL, Japan) at an accelerating voltage of $120 \mathrm{kV}$. Ultrathin sections with the thickness of 60-80 nm were cut from Izod bars perpendicular to the flow direction under cryogenic conditions using a LKB-5 microtome (LKB Co, Switzerland).

Interfacial interaction between GNs and PET was confirmed by dynamic thermal mechanical analysis (DMA, Q800, TA, USA) at $1 \mathrm{~Hz}$ with a heating rate of $2{ }^{\circ} \mathrm{C} \min ^{-1}$ between $40{ }^{\circ} \mathrm{C}$ and $120{ }^{\circ} \mathrm{C}$ under the protection of nitrogen.

Crystallization behavior and crystallinity of GN/PET composites were characterized by differential scanning calorimetry (DSC, Q10, TA, USA) under $\mathrm{N}_{2}$ atmosphere from $40{ }^{\circ} \mathrm{C}$ to $270{ }^{\circ} \mathrm{C}$ at a heating rate of $10{ }^{\circ} \mathrm{C} \mathrm{min}^{-1}$. The formula for calculating crystallinity is as follows:

$$
X_{\mathrm{c}}=\frac{\Delta H_{\mathrm{m}}-\Delta H_{\mathrm{cc}}}{(1-x) \Delta H_{100}} \times 100 \%
$$

where $\Delta H_{\mathrm{m}}$ is melting enthalpy of the samples $\left(\mathrm{J} \mathrm{g}^{-1}\right), \Delta H_{\mathrm{cc}}$ is the enthalpy value of cold crystallization peak, $x$ is the mass fraction of GNs, $\Delta H_{100}$ is the enthalpy value of melting of a $100 \%$ crystalline form of PET $\left(140 \mathrm{~J} \mathrm{~g}^{-1}\right) .{ }^{17}$ 


\section{Results and discussion}

\subsection{Characterization of GNs and modified GNs}

Fig. 1 shows the FT-IR spectra of GNs and modified GNs. In compared with the spectrum of GNs, the spectrum of GNs55 showed a peak for $\mathrm{N}-\mathrm{H}$ stretching vibrations at $1407 \mathrm{~cm}^{-1}$ (Fig. 1(a)). Meanwhile, a weak peak corresponding to epoxy group absorption at $890 \mathrm{~cm}^{-1}$ was observed in the spectrum of GNs560 (Fig. 1(b)). An obvious peak for $\mathrm{C}=\mathrm{O}$ stretching vibration at $1713 \mathrm{~cm}^{-1}$ was seen in the spectrum of GNs570 (Fig. 1(c)). These peaks indicated that KH550, KH560 and KH570 had been respectively grafted onto the surface of GNs. ${ }^{8,18,19}$ Moreover, a relatively strong $\mathrm{Si}-\mathrm{O}-\mathrm{C}$ absorption peak at $1100 \mathrm{~cm}^{-1}$ in the spectra of modified GNs, signified that silanol groups of silane coupling agents had reacted with the hydroxy groups of GNs. This further confirmed that silane coupling agents were successfully grafted onto the surface of GNs in $\mathrm{Sc}-\mathrm{CO}_{2}$ medium.
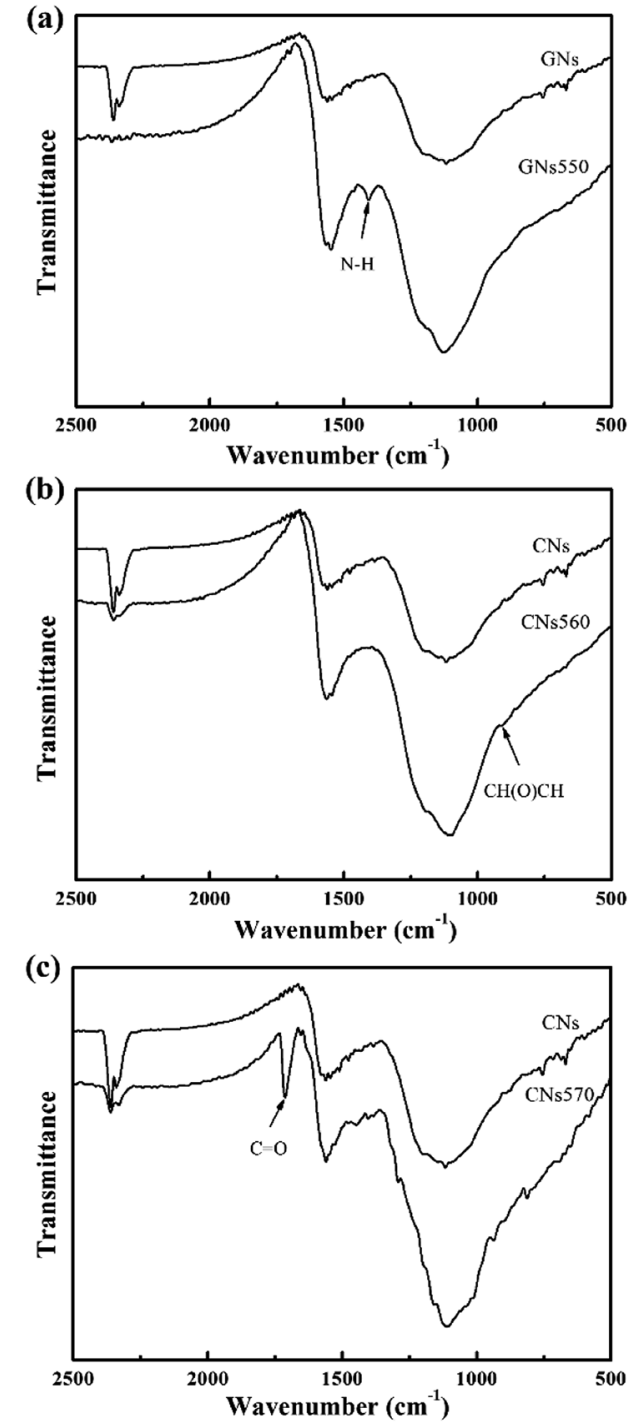

Fig. 1 FTIR spectra: (a) curves of GNs and GNs550; (b) curves of GNs and GNs560; (c) curves of GNs and GNs570.
SEM was employed to observe morphologies of GNs and modified GNs and the results were shown in Fig. 2. It was evident that the size of modified GNs tactoids was significantly reduced compared with those of GNs, and the GNs570 showed the smallest tactoid size (Fig. 2(d)). It is noteworthy that the morphologies of modified GNs were also quite different from that of GNs. Obviously, pristine GNs appeared a tight tactoids (Fig. 2(a)), composed of some stacked platelets held together by van der Waals forces. Differently, the morphology of modified GNs showed a fluffy structure and the thickness of the tactoids was thinner, signifying that $\mathrm{Sc}-\mathrm{CO}_{2}$ fluid along with silane coupling agent could permeate through the laminates of GNs, inducing exfoliation of stacked platelets of GNs.

Fig. 3 showed EDX spectral curves of GNs and modified GNs. It is seen that the curve of GNs only showed peaks of $\mathrm{O}$ and $\mathrm{C}$ elements. Excitingly, all of the curves of modified GNs appeared the peak of Si element, further demonstrating that silane coupling agents had been grafted onto the surface of GNs.

Moreover, the grafting ratios of silane coupling agents onto the surfaces of GNs were respectively studied by TG analyzer. GNs showed very less weight loss from $200{ }^{\circ} \mathrm{C}$ to $700{ }^{\circ} \mathrm{C}$ (Fig. 4). However, for GNs550, GNs560, and GNs570, the weight loss decreased sharply from $200{ }^{\circ} \mathrm{C}$ to $700{ }^{\circ} \mathrm{C}$ (Fig. 4), which could be attributed to the pyrolysis of the silane coupling agents. Based on the results of weight loss from $200{ }^{\circ} \mathrm{C}$ to $700{ }^{\circ} \mathrm{C}$, the grafting ratios of GNs550, GNs560, and GNs570 were calculated to be about $2.91 \%, 2.12 \%$, and $3.57 \%$, respectively.
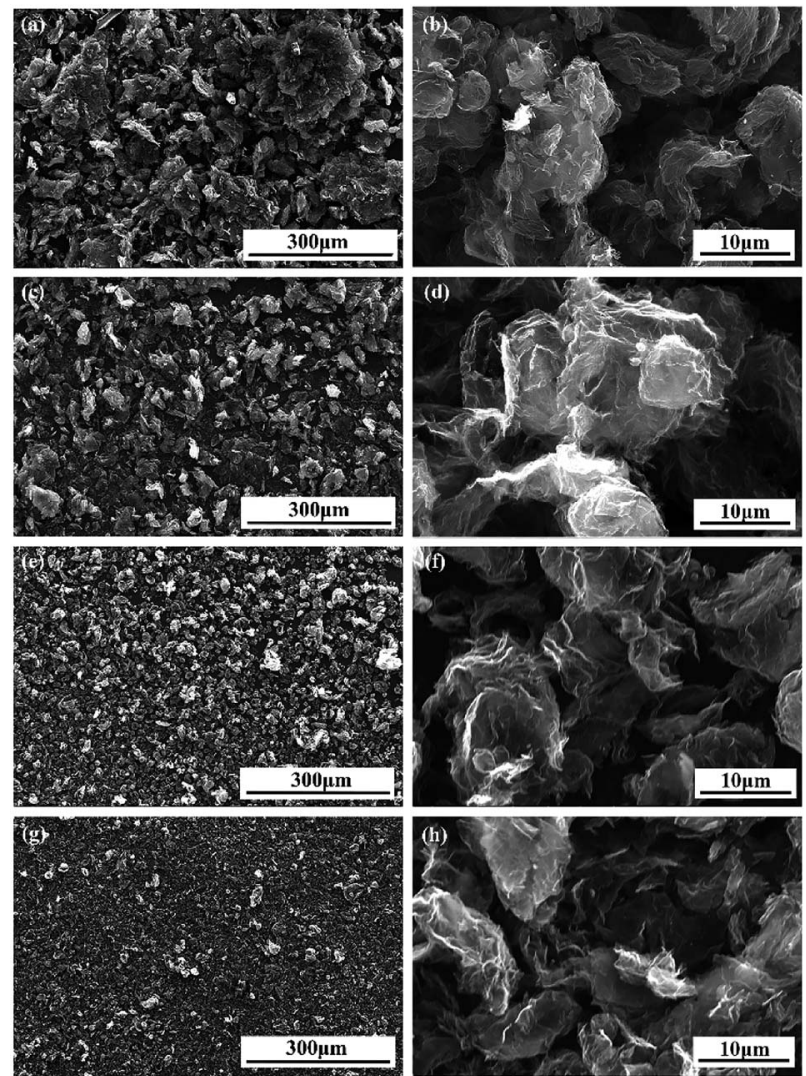

Fig. 2 SEM micrographs: (a and b) GNs; (c and d) GNs550; (e and f) GNs560, (g and h) GNs570. 


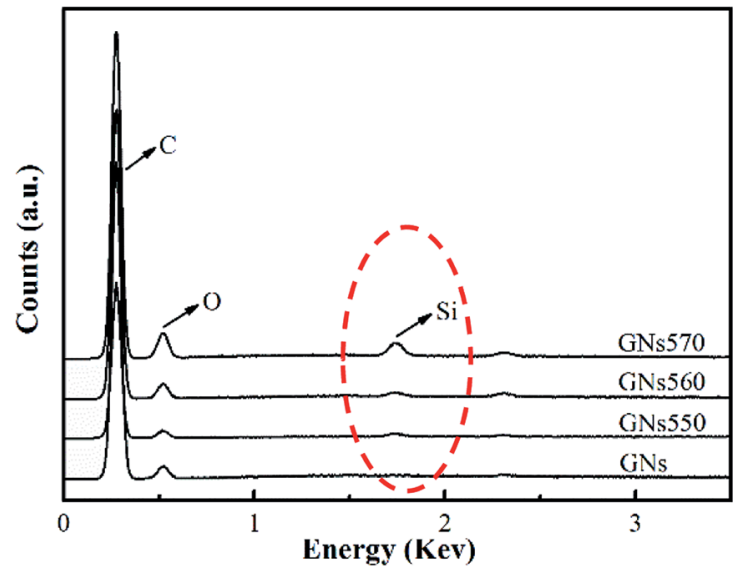

Fig. 3 EDX spectra of GNs, GNs550, GNs560, and GNs570.

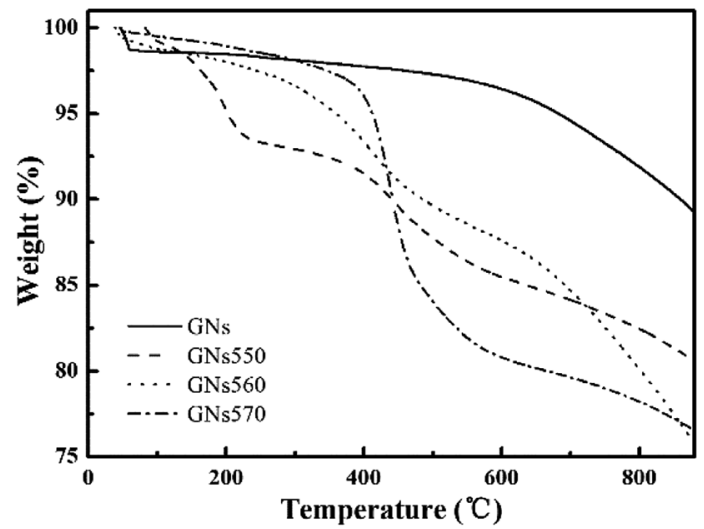

Fig. 4 TG curves of GNs, GNs550, GNs560 and GNs570 in $\mathrm{N}_{2}$ atmosphere.

Based on above analyses, it can be concluded that $\mathrm{Sc}-\mathrm{CO}_{2}$ processing was a simple and effective technology to modify GNs with silane coupling agents, the modified process and modification mechanism was illustrated in Fig. 5(a) and (b).

\subsection{Barrier properties of GN/PET composite films}

To study effect of modified GNs on barrier properties of GN/PET composite films, pristine GNs was also incorporated into PET to obtain GN/PET composite film using the multistage stretching extrusion technique. Fig. 6 showed OTR and WVTR values of neat PET and the prepared GN/PET composites. Apparently, OTR of PET film was effectively reduced on addition of less amount of pristine GNs. More specifically, there was more than $38 \%$ reduction in OTR (from $6.96 \mathrm{~cm}^{3}$ per $\mathrm{m}^{2}$ per day to 4.31 $\mathrm{cm}^{3}$ per $\mathrm{m}^{2}$ per day) on addition of only $0.03 \%$ GNs. As expected, the composite films prepared by modified GNs showed further decrease in OTR with the exception of that prepared by GNs560. It is seen that OTR of the composites prepared by GNs550 and GNs570 was separately decreased by about $58.04 \%$ and $51.00 \%$.

In contrast, the WVTR of PET film remained almost unchanged with addition of GNs. However, that was significantly decreased with addition of GNs560 and GNs550 (as shown in Fig. 6). It is noteworthy that the reduction was approximately about $90.08 \%$ for the composite prepared by GNs560. Unexpectedly, the WVTR of GNs570/PET composite films showed abnormal increase, as shown in Fig. 6.

\subsection{Structures of GN/PET composite films}

To gain an in-depth understanding of the effect of modified GNs on the barrier properties of GN/PET composite films, a series of structural characterizations were carried out. It is known that the tortuous path effect caused by layered nanoparticles is a key factor in improving the gas barrier properties of layered nanoparticle/polymer composites. ${ }^{20}$ Therefore, morphology of the layered nanoparticles (exfoliation, dispersion, and orientation relative to the diffusion direction) and the interfacial interaction between the particles and polymer matrix had showed prominent effect on the barrier properties of the composites. In this study, TEM was employed to study the morphologies of GNs in the composites and the results were shown in Fig. 7, wherein the white regions denote PET matrix and the dark flakes represent aggregates or platelets of GNs. In Fig. 7(a) pristine GNs appeared oriented aggregates in PET matrix with the size of $0.05-0.12 \mu \mathrm{m}$. Contrastingly, the modified GNs dispersed in PET matrix as exfoliated platelets (Fig. 7(b-d)), implying that PET chains could permeate into the laminates of modified GNs during melt processing.

In comparison with dispersion, the interfacial interaction between nanoparticles and polymer matrix was more important for improving barrier property of the nanocomposites. ${ }^{\mathbf{6} 20}$ In this study, DMA was carried out to determine the interfacial adhesion of the prepared composites and the results were shown in Fig. 8. Since the area under the DMA loss modulus was a parameter associated with phase interaction, the enveloped area $(S)$ reflected the interfacial adhesion between nanoparticles and polymer matrix, a larger $S$ value revealed stronger interaction between the nanoparticles and polymer matrix. ${ }^{21}$ Moreover, the interface action parameter $b$ also reflected the interfacial adhesion strength between GNs and PET matrix, and that parameter $b$ could be calculated by the following formula: ${ }^{22}$

$$
\frac{E_{\mathrm{c}}^{\prime \prime}}{E_{\mathrm{o}}^{\prime \prime}}=\left(1-b v_{\mathrm{f}}\right)^{-1}
$$

where, $E_{\mathrm{o}}^{\prime \prime}$ and $E_{\mathrm{c}}^{\prime \prime}$ are the loss moduli of composites and neat PET, respectively, and $v_{\mathrm{f}}$ is the volume fraction of GNs in the composite, which was same for all samples.

The parameter $b$ calculated from eqn (4) and the value of $S$ calculated from DMA loss moduli curves were summarized in Table 1. As expected, all of the modified GN/PET composites showed increased loss modulus and $S$ as well as $b v_{\mathrm{f}}$. Accordingly, modified GNs showed relatively stronger interaction with PET matrix as compared to that of pristine GNs. Different modified GNs showed different interaction capacity with PET matrix, and GNs modified by KH560 displayed the strongest interaction with PET matrix. In addition, the improved storage moduli of all of the modified GN/PET composites further confirmed the above results (as shown in Fig. 8(a)). 

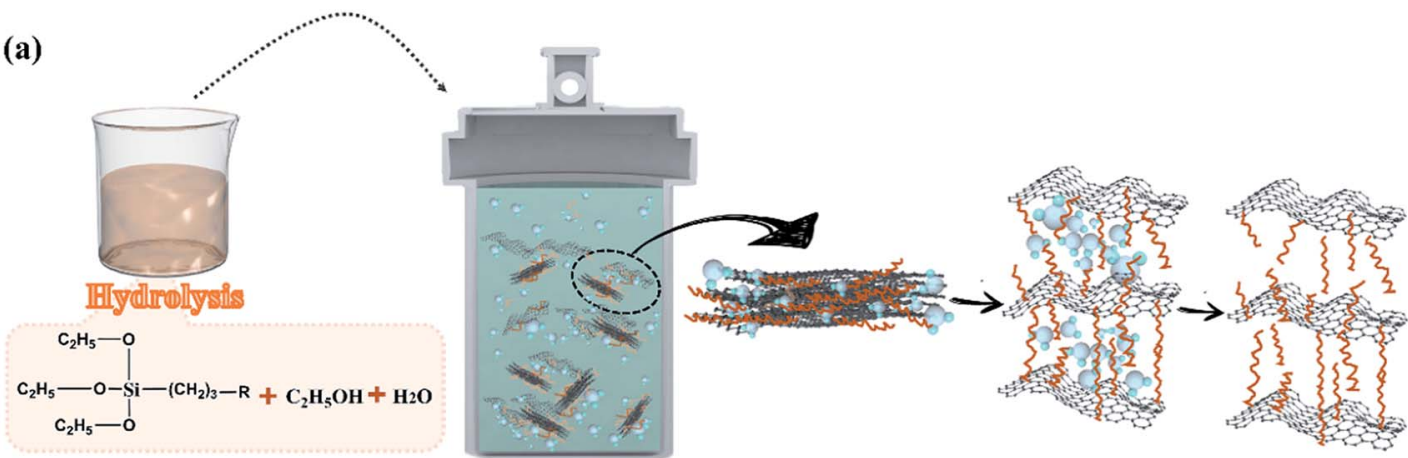

(b)

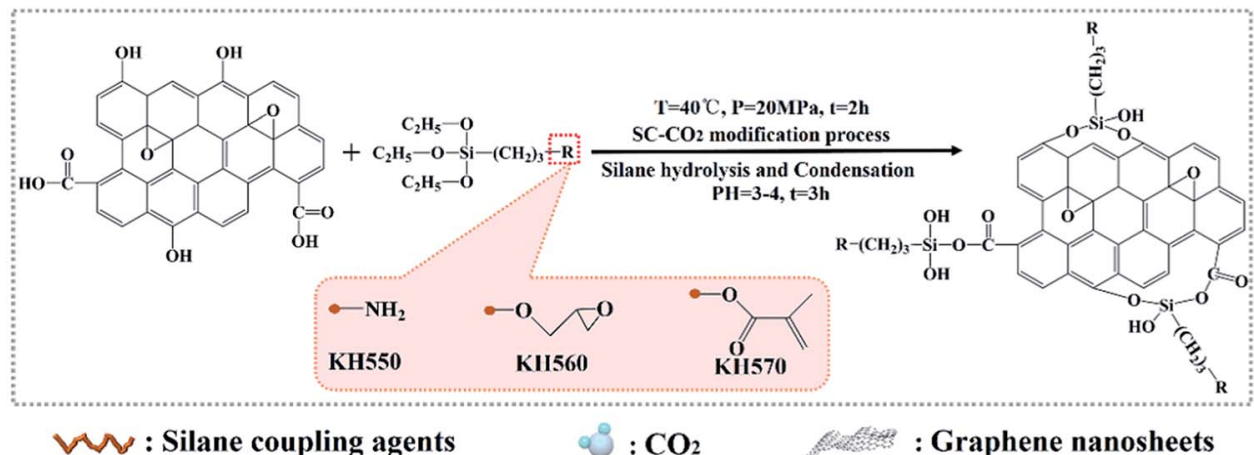

Fig. 5 Schematic drawing of GNs surface modification.

Moreover, crystallinity also showed a great influence on the barrier properties of composites. ${ }^{6,723}$ Therefore, DSC analysis was conducted to study the crystallization behaviors of prepared composites. Fig. 9 showed DSC curves of neat PET and GN/PET composites and the results were shown in Table 2. Obviously, crystallization temperatures $\left(T_{\mathrm{c}}\right)$ of GN/PET composites were increased by about $10{ }^{\circ} \mathrm{C}$, as compared with that of neat PET. This suggested that GNs showed prominent heterogeneous nucleation effect on PET. Meanwhile the initial slope of crystallization peak $\left(S_{\mathrm{i}}\right)$ increased obviously with addition of GNs, indicating that the initial nucleation rate of GN/PET composites was enhanced greatly, especially those composites prepared by modified GNs. Similarly, decrease in supercooling temperature $(\Delta T)$ of GN/PET composites also indicated that the crystallization capacity of the composites increased with addition of GNs. Surprisingly, the crystallinity of all of the prepared GN/PET

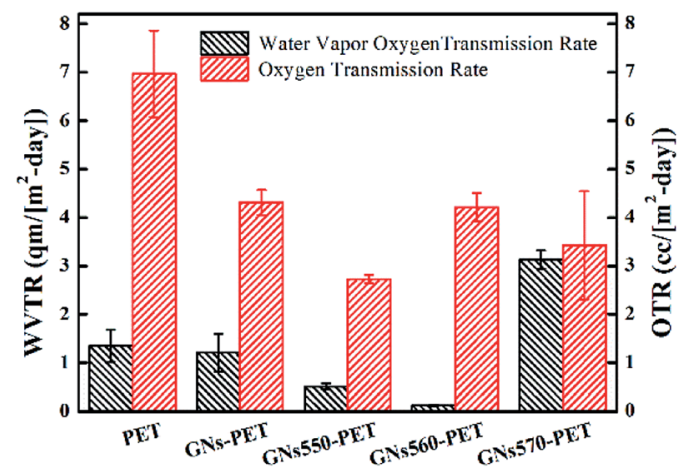

Fig. 6 OTR and WVTR of neat PET and GN/PET composite films. composites was the same as that of neat PET, suggesting that crystallinity of GN/PET composites should make no contribution to the improvement of barrier properties.

\subsection{Proposed barrier mechanism of GN/PET composites}

Based on above DSC results, it can be deduced that the tortuous path effect caused by GNs was the mainly responsible for the barrier properties of the prepared GN/PET composites. ${ }^{6,21,24}$ Therefore, the enhanced barrier property of pristine GN/PET
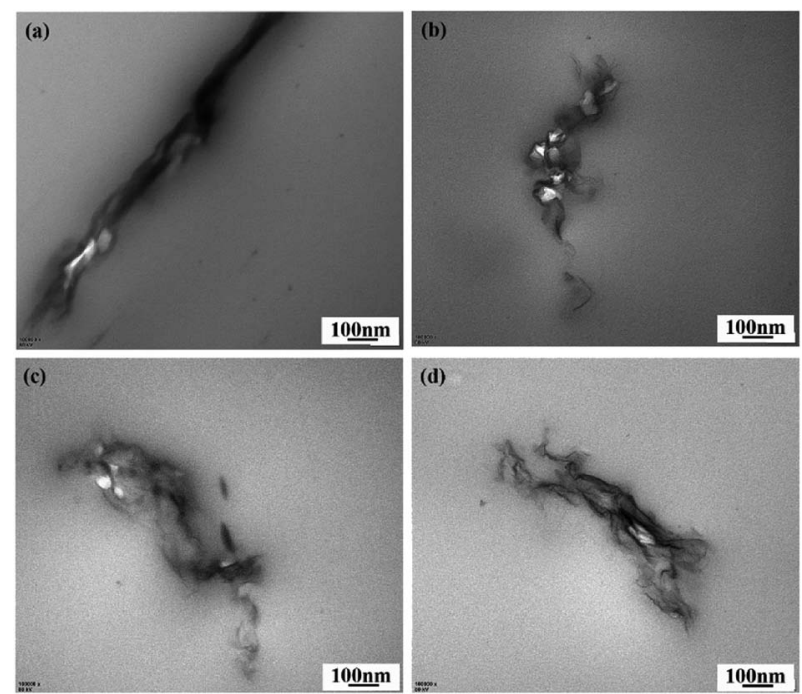

Fig. 7 TEM images of GN/PET composites: (a) GN/PET, (b) GNs550/ PET, (c) GNs560/PET, (d) GNs570/PET. 

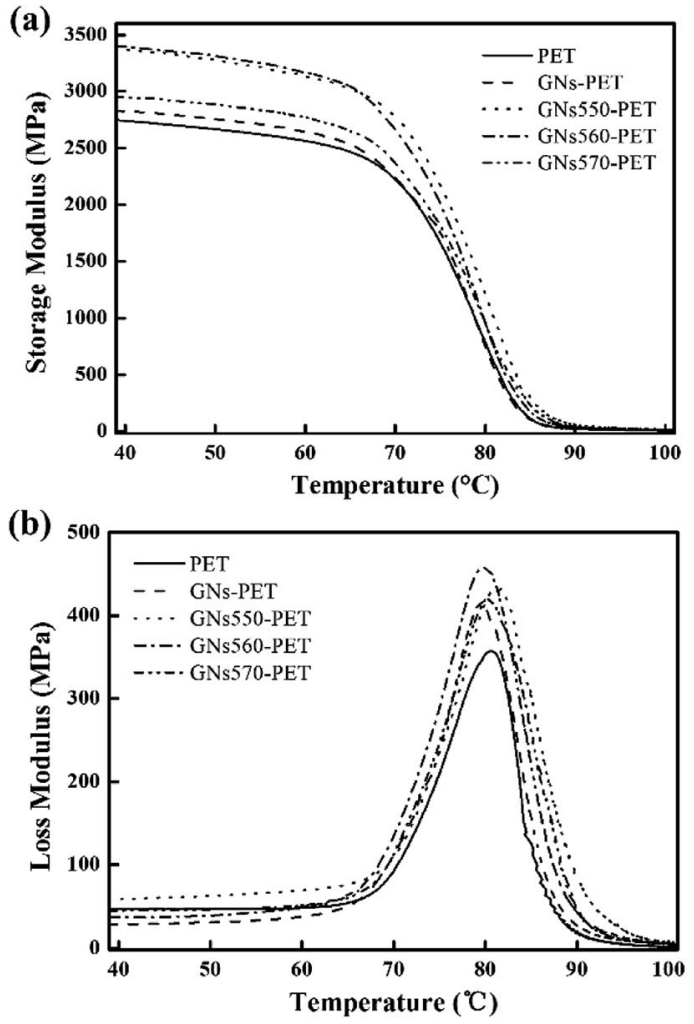

Fig. 8 Dependence of (a) storage modulus $\left(E^{\prime}\right)$, (b) loss modulus $\left(E^{\prime \prime}\right)$ on temperature in case of neat PET and GN/PET composite films.

composites was attributed to the oriented dispersion of GNs aggregates, as confirmed by TEM micrographs (Fig. 7), which could extend the permeation pathway for oxygen and water vapor. While the greater reduction of OTR than that of WVTR was probably due to the relatively poor interfacial interaction of GNs and PET. It is deduced that some interfacial defects might present in the pristine GN/PET composites for the worse interfacial adhesion as confirmed by DMA (see in Fig. 8 and Table 1). Because the kinetic diameter of $\mathrm{H}_{2} \mathrm{O}(0.29 \mathrm{~nm})$ was smaller than that of $\mathrm{O}_{2}(0.35 \mathrm{~nm}),{ }^{25}$ so that much $\mathrm{H}_{2} \mathrm{O}$ molecules could penetrate through the interfacial defects as compared to $\mathrm{O}_{2}$ molecules, leading to the differences in reduction of OTR and WVTR values. ${ }^{26}$

In addition, in case of composites prepared from modified GNs, the improvement in dispersion was not always consistent with decreased OTR and WVTR values as shown in Fig. 6, indicating that the barrier properties of modified GN/PET composites were mainly dependent on the interfacial interaction of the composites. According to DMA results that the interfacial
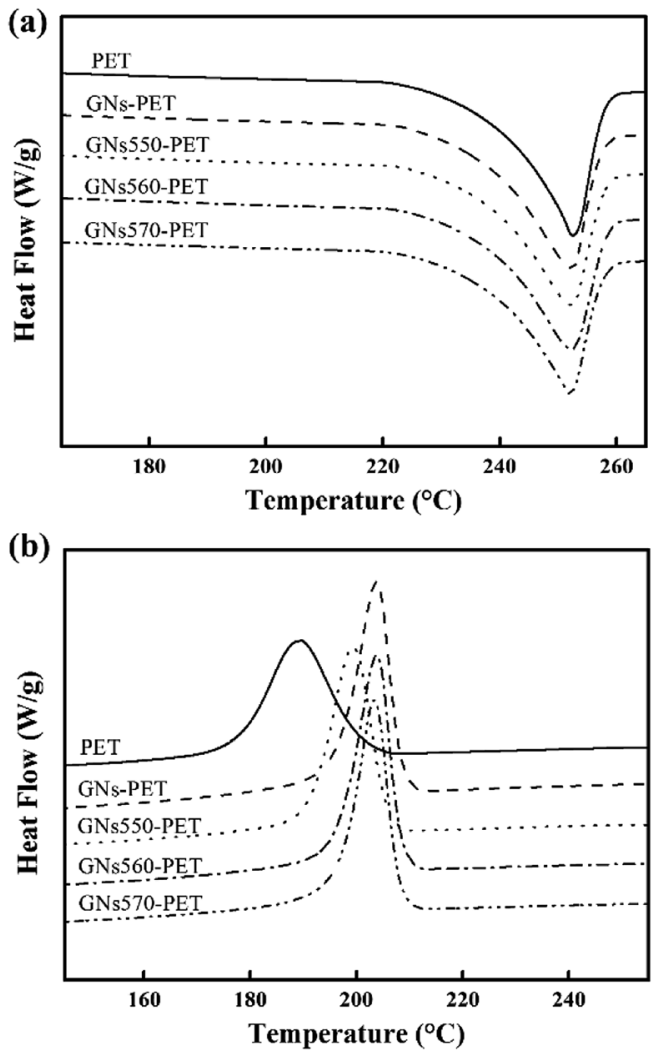

Fig. 9 DSC curves: (a) melting curves; (b) crystallization curves.

interaction of GNs550/PET and GNs560/PET composites was stronger than that of GNs570/PET composite (see in Table 1), attributing to the amino group of KH550 and epoxy group of KH560 could react with the terminal carboxyl and hydroxyl groups on PET chain, while the terminal methyl group and carbon-carbon double bond of KH570 done rarely, implying that more interfacial defects were created in GNs570/PET composites as compared to the composites prepared from GNs550 and GNs560 during the multistage stretching extrusion process. Based on above analysis, it is inferred that the further reduction of OTR and WVTR of the modified GN/PET composites was due to the improved dispersion structure and interfacial adhesion (see in Fig. 7 and Table 1). The lowest OTR of GNs550/PET composite was ascribed to the well interfacial adhesion as compared to GNs570/PET composite, while the almost unchanged OTR of GNs560/PET composite might due to the affinity of oxygen molecules to hydroxyl groups produced by reaction of epoxy group with the terminal hydroxyl and carboxyl groups on PET molecular chain, ${ }^{27}$ balancing the positive effect of interfacial adhesion.

Table 1 Parameters calculated from DMA analysis

\begin{tabular}{llcrr}
\hline specimen & PET & GN/PET & GNs550/PET & GNs560/PET \\
\hline$S(\mathrm{MPa}$ min $)$ & 4464.72 & 5916.62 & 6400.57 & 6417.24 \\
$E^{\prime \prime}(\mathrm{MPa})$ & 356.90 & 418.90 & 434.80 & 459.40 \\
$E_{\mathrm{o}}^{\prime \prime} / E_{\mathrm{c}}^{\prime \prime}$ & - & 1.17 & 1.22 & 1.29 \\
$b v_{\mathrm{f}}$ & - & 0.148 & 0.179 & 0.223
\end{tabular}


Table 2 DSC results

\begin{tabular}{|c|c|c|c|c|c|}
\hline Specimen & PET & GN/PET & GNs550/PET & GNs560/PET & GNs570/PET \\
\hline$T_{\mathrm{cm}}$ & 126.23 & 126.90 & 131.52 & 129.95 & 126.78 \\
\hline$T_{\mathrm{m}}$ & 252.52 & 252.47 & 252.31 & 252.23 & 252.10 \\
\hline$X_{\mathrm{c}}$ & 0.1345 & 0.1309 & 0.1310 & 0.1344 & 0.1453 \\
\hline$T_{\mathrm{c}}$ & 189.65 & 203.67 & 199.22 & 203.70 & 203.32 \\
\hline$S_{\mathrm{i}}$ & 0.0443 & 0.1908 & 0.1355 & 0.2025 & 0.2019 \\
\hline
\end{tabular}

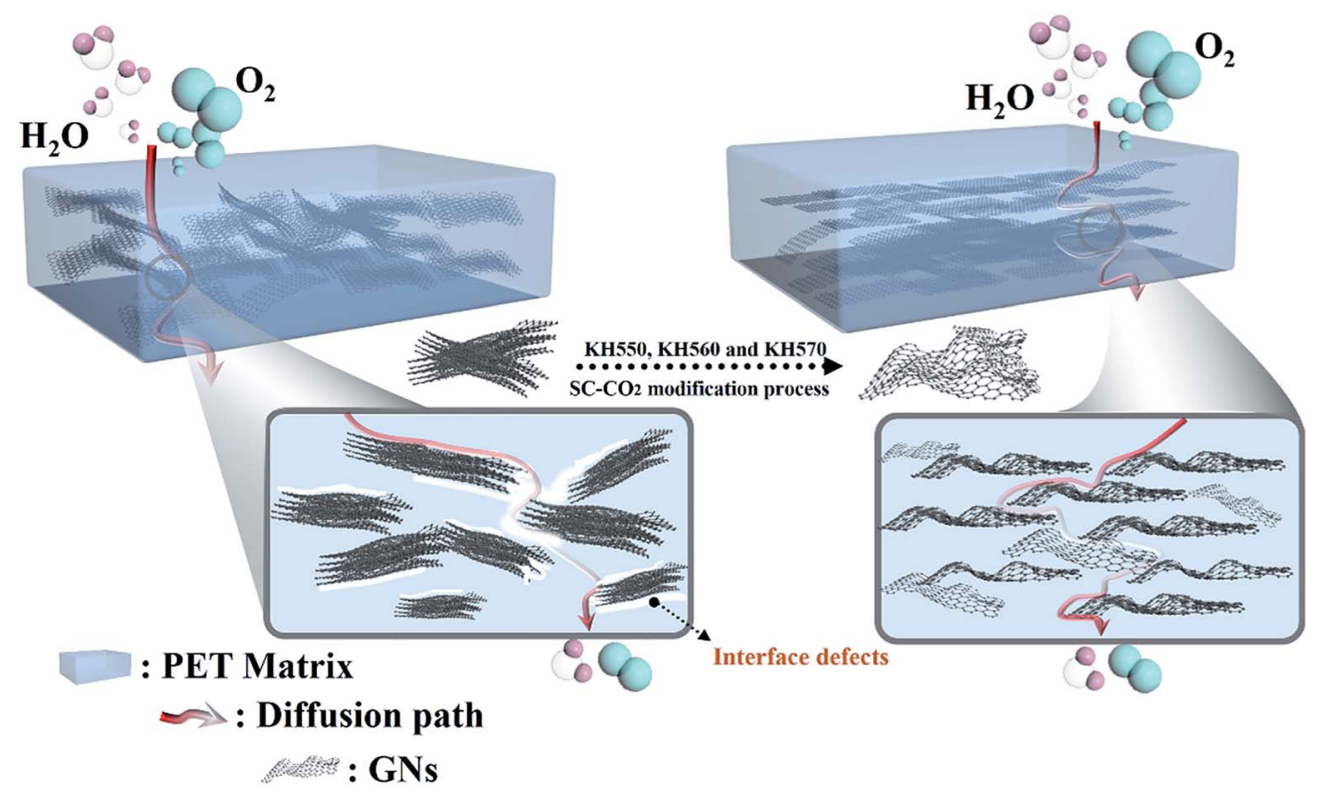

Fig. 10 Schematic diagram of possible barrier mechanism of GN/PET composite films.

Furthermore, the extraordinarily high WVTR of GNs570/PET composite was mainly due to the relatively more interfacial defects induced by the relatively worse interfacial adhesion (see in Table 1), making the water vapor molecules could permeate through the interface of GNs570 and PET, leading to the increase of WVTR. While the decreased OTR of GNs570/PET composite suggested that the size of the interfacial defects was smaller than that of kinetic diameter $(0.35 \mathrm{~nm})$ of oxygen molecules, resulting in the differences between OTR and WVTR. $^{26}$

In conclusion, the tortuous path effect caused by GNs and the interfacial interaction were mainly responsible for the barrier properties of GN/PET composites. It was inferred that the barrier properties of GN/polymer composites were attributed to not only the tortuous path effect caused by GNs itself and the interfacial interaction, but also the affinity of binding bonds between GNs and polymer to the gas molecules. Eventually, barrier mechanism of GN/PET and modified GN/PET has been displayed and illustrated schematically in Fig. 10.

\section{Conclusions}

In summary, a simple and eco-friendly strategy of $\mathrm{Sc}^{-\mathrm{CO}_{2}}$ process was employed to modify GNs with different silane coupling agents, and effects of the different modified GNs on oxygen barrier property and water vapor barrier property of the composite films were studied. FT-IR results indicated that silane coupling agents has been successfully grafted onto GNs surface. SEM investigations showed that GNs modified with silane coupling agent by $\mathrm{Sc}-\mathrm{CO}_{2}$ processing showed a fluffy structure and a finer size of tactoids. It is demonstrated that Sc- $\mathrm{CO}_{2}$ strategy could tune not only the surface property of GNs, but also the morphology of GNs. While barrier property studies indicated that modified GNs could significantly enhance oxygen barrier and water vapor barrier properties of the composite films. It is found that oxygen barrier properties of GNs550/PET composite films increased about 58.04\%. While the water vapor barrier property of GNs560/PET composite film was enhanced about $90.08 \%$. It is proved that the enhanced barrier properties were attributed to the tortuous path effect caused by GNs and the improved interfacial adhesion as well as the weak affinity of binding bonds to the gas molecules. It suggested that a suitable modifier must be carefully chosen based on practical requirements, due to the different physical property of oxygen gas and water vapor. We believed that this study provided a better guidance for preparing layered nanoparticle/polymer composites with outstanding barrier properties. 


\section{Conflicts of interest}

There are no conflicts to declare.

\section{Acknowledgements}

The authors would like to acknowledge the financially supported from Natural Science Foundation of China (Grant: 51303032, 51473186), the Major Special Projects of Guizhou province (Grant: (2015)6005), the Science and Technology Support Project of Guizhou (Grant: (2019)2836).

\section{References}

1 Y. Z. Yoo, J. Y. Na, Y. S. Choi, Y. J. Lim, J. H. Kim, Y. B. Kim, J. H. Kim, S. K. Kim and T. Y. Seong, Small, 2018, 14, 1800056.

2 P. Xiong, Y. Liu, D. Tao, C. Ping, H. Wang and D. Yu, Org. Electron., 2018, 58, 18-24.

3 A. A. Abareen, H. A. Ustami, H. Harel and G. Marom, J. Appl. Polym. Sci., 2013, 128, 1534-1539.

4 H. Kim, Y. Miura and C. W. Macosko, Chem. Mater., 2010, 22, 3441-3450.

5 O. C. Compton, K. Soyoung, P. Cynthia, J. M. Torkelson and S. B. T. Nguyen, Adv. Mater., 2010, 22, 4759-4763.

6 H. D. Huang, P. G. Ren, J. Z. Xu, X. U. Ling, G. J. Zhong and B. S. Hsiao, J. Membr. Sci., 2014, 464, 110-118.

7 J. Teng, B. Niu, L. Q. Zhang, J. Xu, L. Xu, Z. Yan, J. H. Tang, G. J. Zhong and Z. M. Li, RSC Adv., 2016, 6, 68072-68080.

8 P. Xu, X. Yan, P. Cong, X. Zhu and D. Li, Compos. Interfaces, 2016, 24, 1-14.

9 S. Pourhashem, A. Rashidi, M. R. Vaezi and M. R. Bagherzadeh, Surf. Coat. Technol., 2017, 317, 1-9.

10 Y. Wang, Z. Chen, Z. Wu, Y. Li, W. Yang and Y. Li, Langmuir, 2018, 34, 7797-7804.
11 S. S. Xu, Q. Xu, N. Wang, Z. M. Chen, Q. G. Tian, H. X. Yang and K. X. Wang, Chem. Mater., 2015, 27, 3262-3272.

12 W. T. He, X. Chen, S. H. Qin, J. Yu, M. He, Y. Yao and Q. Zhang, Adv. Powder Technol., 2014, 25, 1786-1792.

13 Q. Tian, Y. T. Qi, S. H. Qin, F. Z. Wu, L. J. Long, G. M. Xu and X. G. Yin, J. Macromol. Sci., Part B: Phys., 2017, 56, 474-492.

14 Q. Tian, S. H. Qin, L. J. Long, Y. H. Jiang, R. F. Zhou, R. Zhou, W. T. He and G. M. Xu, J. Vinyl Addit. Technol., 2015, 22, 423432.

15 W. T. He, Y. Yong, S. H. Qin, J. Yu, M. He, C. Xu and G. M. Xu, J. Macromol. Sci., Part B: Phys., 2016, 55, 445-456.

16 L. Fan, H. Yu, S. Yang, H. M. Sun, L. Jiang, S. Y. Guo, H. Wu, J. B. Shen, C. Rong and X. Ying, Polymer, 2016, 82, 274-284.

17 Y. F. Lin, R. Tyler, H. Sun, K. Y. Shi and D. A. Schiraldi, Polymer, 2017, 127, 236-240.

18 J. Li, J. C. Cui, J. Y. Yang, M. Yuan, H. X. Qiu and J. H. Yang, Prog. Org. Coat., 2016, 99, 443-451.

19 P. Klunbud, P. Suktha and M. Sawangphruk, J. Appl. Polym. Sci., 2016, 133, 44382.

20 H. D. Huang, S. Y. Zhou, D. Zhou, P. G. Ren, J. Z. Xu, X. Ji and Z. M. Li, Ind. Eng. Chem. Res., 2016, 55, 9544-9554.

21 W. H. Ruan, M. Z. Rong and M. Q. Zhang, Mater. Res. Appl., 2010, 04, 736-739.

22 M. Sumita, H. Tsukihi, K. Miyasaka and K. Ishikawa, J. Appl. Polym. Sci., 2010, 29, 1523-1530.

23 S. Zhou, J. Chen, X. Li, X. Ji, G. Zhong and Z. Li, RSC Adv., 2016, 6, 2530-2536.

24 H. G. Kim, E. H. Kim and S. S. Kim, Macromol. Res., 2018, 26, 1-5.

25 B. D. Freeman, Macromolecules, 1999, 32, 375-380.

26 A. P. Roberts, B. M. Henry, A. P. Sutton, C. R. M. Grovenor, G. A. D. Briggs, T. Miyamoto, M. Kano, Y. Tsukahara and M. Yanaka, J. Membr. Sci., 2002, 208, 75-88.

27 Y. L. Zhang, X. X. Jing, K. G. Jing, L. P. Chang and W. R. Bao, Appl. Surf. Sci., 2015, 324, 90-98. 